Nonlin. Processes Geophys., 25, 659-669, 2018

https://doi.org/10.5194/npg-25-659-2018

(C) Author(s) 2018. This work is distributed under

the Creative Commons Attribution 4.0 License.

\title{
Internal waves in marginally stable abyssal stratified flows
}

\author{
Nikolay Makarenko ${ }^{1,2}$, Janna Maltseva ${ }^{1,2}$, Eugene Morozov $^{3}$, Roman Tarakanov $^{3}$, and Kseniya Ivanova ${ }^{4}$ \\ ${ }^{1}$ Lavrentyev Institute of Hydrodynamics, 6300090, Novosibirsk, Russia \\ ${ }^{2}$ Novosibirsk State University, Department of Mechanics and Mathematics, 630090, Novosibirsk, Russia \\ ${ }^{3}$ Shirshov Institute of Oceanology, 117997, Moscow, Russia \\ ${ }^{4}$ Aix-Marseille University, Department of Mechanics, Marseille 06, France
}

Correspondence: Nikolay Makarenko (makarenko@hydro.nsc.ru)

Received: 11 January 2018 - Discussion started: 26 January 2018

Revised: 13 June 2018 - Accepted: 10 July 2018 - Published: 5 September 2018

\begin{abstract}
The problem on internal waves in a weakly stratified two-layer fluid is studied semi-analytically. We discuss the 2.5-layer fluid flows with exponential stratification of both layers. The long-wave model describing travelling waves is constructed by means of a scaling procedure with a small Boussinesq parameter. It is demonstrated that solitarywave regimes can be affected by the Kelvin-Helmholtz instability arising due to interfacial velocity shear in upstream flow.
\end{abstract}

\section{Introduction}

In this paper, we consider an analytical model of internal solitary waves in a two-layer fluid with the density continuously increasing with depth in both layers. This model is a development of non-linear two-layer models previously suggested by Ovsyannikov (1985), Miyata (1985) and Choi and Camassa (1999), as well as the latest 2.5-layer models considered by Voronovich (2003) and Makarenko and Maltseva (2008, 2009a, b). Two-layer approximation is a standard model of a sharp pycnocline in a stratified fluid with constant densities in each layer, but which is discontinuous at the interface. Correspondingly, the 2.5-layer model takes into account a slight density gradient in stratified layers which is comparable with the density jump at the interface. In all these cases, internal solitary waves can be described in closed form by the solutions resulting from the quadrature

$$
\left(\frac{\mathrm{d} \eta}{\mathrm{d} x}\right)^{2}=f(\eta)
$$

for stationary wave elevation $\eta(x)$. The simplest version of non-linearity $f$ appears in a two-layer system; hence, it is the rational function $f(\eta)=P(\eta) / Q(\eta)$ where $P$ is a fourthdegree polynomial and $Q$ depends linearly on $\eta$. Equation (1) also appears as a travelling wave equation for non-linear evolution systems similar to the single-layer dispersive GreenNaghdi model (see Choi and Camassa, 1999). These nonlinear dispersive equations can be obtained by means of a long-wave perturbation technique as well as by Whitham's variational method. Several authors noted that solitary-wave solutions of such approximate models are in good agreement with the numerical solutions of fully non-linear Euler equations for a perfect two-layer fluid. In this context, Camassa and Tiron (2011) also compared numerical travelling wave solutions, supported by smooth stratification, with the known explicit solitary-wave solutions in order to optimize a twolayer model of the Euler system with smooth stratification.

We apply the method of derivation involving asymptotic analysis of the non-linear Dubreil-Jacotin-Long equation that results from fully non-linear Euler equations of stratified fluid. The long-wave scaling procedure uses a small Boussinesq parameter which characterizes slightly increasing density in the layers and a small density jump at their interface. This method combines the approaches applied formerly to a pure two-fluid system with the perturbation technique discussed for the first time by Long (1965) and developed by Benney and Ko (1978) for a continuous stratification. The parametric range of a solitary wave is considered in the framework of the constructed mathematical model. It is demonstrated that these wave regimes can approach the parametric domain of the Kelvin-Helmholtz instability. The stability of solitary travelling-wave solutions of 


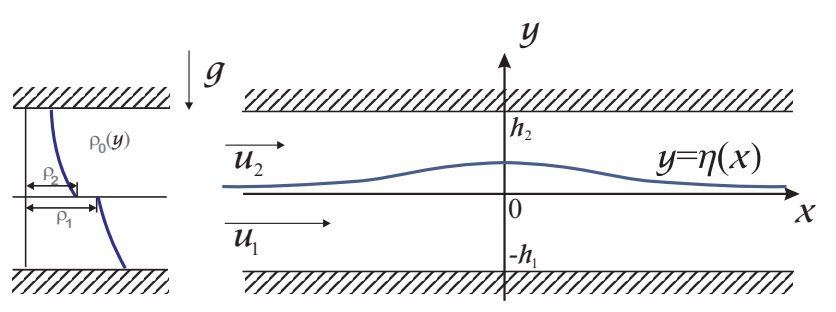

Figure 1. Scheme of the flow.

the Euler equations for continuously stratified, near two-layer fluids was studied numerically and analytically by Almgren et al. (2012). They demonstrated that the wave-induced shear can locally reach unstable configurations and give rise to local convective instability. This is in good qualitative agreement with the laboratory experiments performed by Grue et al. (2000). It seems that such a marginal stability of long internal waves could explain the formation mechanism of very long billow trains in abyssal flows observed by Van Haren et al. (2014).

\section{Basic equations}

We consider a 2-D motion of inviscid two-layer fluid which is weakly stratified due to gravity in both layers. The fully non-linear Euler equations describing the flow are

$$
\begin{aligned}
& \rho\left(u_{t}+u u_{x}+v u_{y}\right)+p_{x}=0, \\
& \rho\left(v_{t}+u v_{x}+v v_{y}\right)+p_{y}=-\rho g, \\
& \rho_{t}+u \rho_{x}+v \rho_{y}=0 \\
& u_{x}+v_{y}=0
\end{aligned}
$$

where $\rho$ is the fluid density, $(u, v)$ is the fluid velocity, $p$ is the pressure and $g$ is the gravity acceleration. We assume that the flow domain is bounded by the flat bottom $y=-h_{1}$ and the rigid lid $y=h_{2}$ (see Fig. 1), with the boundary condition

$v=\left.0\right|_{y=-h_{1}, y=h_{2}}$.

The layers are separated by the interface $y=\eta(x, t)$ with the equilibrium level at $y=0$. Non-linear kinematic and dynamic boundary conditions at this interface are

$\eta_{t}+u \eta_{x}=\left.v\right|_{y=\eta}, \quad[p]=\left.0\right|_{y=\eta}$,

where the square brackets denote the discontinuity jump at the interface between the layers. Non-disturbed parallel flow has no vertical velocity and elevation (i.e. $v=0, \eta=0$ ), but the horizontal velocity $u=u_{0}(y)$ may be piece-wise constant:

$u_{0}(y)=\left\{\begin{array}{lc}u_{1} & \left(-h_{1}<y<0\right), \\ u_{2} & \left(0<y<h_{2}\right) .\end{array}\right.$
In this stationary case, the fluid density $\rho=\rho_{0}(y)$ and pressure $p=p_{0}(y)$ should be coupled by the hydrostatic equation $\mathrm{d} p_{0} / \mathrm{d} y=g \rho_{0}$. We consider the density profile depending exponentially on height,

$\rho_{0}(y)= \begin{cases}\rho_{1} \exp \left(-N_{1}^{2} y / g\right) & \left(-h_{1}<y<0\right), \\ \rho_{2} \exp \left(-N_{2}^{2} y / g\right) & \left(0<y<h_{2}\right),\end{cases}$

where $N_{j}=$ const is the Brunt-Väisälä frequency in the $j$ th layer, and constant densities $\rho_{1}$ and $\rho_{2}$ are related as $\rho_{2}<\rho_{1}$. The special case $N_{j}=0(j=1,2)$ gives a familiar two-fluid system with piece-wise constant density $\rho=\rho_{j}$ in the $j$ th layer.

Further we consider a steady non-uniform flow; hence, we have $\eta_{t}=0$ and $u_{t}=v_{t}=\rho_{t}=0$ in Eqs. (2)-(4). We introduce the stream function $\psi$ by standard formulae $u=$ $\psi_{y}, v=-\psi_{x}$; hence, the mass conservation implies the dependence $\rho=\rho(\psi)$, and pressure $p$ can be found from the Bernoulli equation

$\frac{1}{2}|\nabla \psi|^{2}+\frac{1}{\rho(\psi)} p+g y=b(\psi)$.

Seeking solitary-wave solutions, we require that the upstream velocity of the fluid $(u, v)$ tend to $\left(u_{j}, 0\right)$ as $x \rightarrow-\infty$. In this case, boundary conditions (6) transform to the conditions for the stream function as

$\psi=-\left.u_{1} h_{1}\right|_{y=-h_{1}}, \quad \psi=\left.0\right|_{y=\eta}, \quad \psi=\left.u_{2} h_{2}\right|_{y=h_{2}}$.

It is known (Yih, 1980) that systems (2)-(5) can be reduced in a stationary case to the non-linear Dubreil-Jacotin-Long (DJL) equation for the stream function

$\rho(\psi) \nabla^{2} \psi+\rho^{\prime}(\psi)\left(g y+\frac{1}{2}|\nabla \psi|^{2}\right)=H^{\prime}(\psi)$.

Here, the function $H(\psi)=\rho(\psi) b(\psi)$ involves the Bernoulli function $b(\psi)$ and the density function $\rho(\psi)$, so that $H$ is specified by the upstream condition. More exactly, the density function is determined by the relation $\rho(\psi)=\rho_{0}\left(\psi / u_{j}\right)$ in the $j$ th layer, and the Bernoulli function $b(\psi)$ is defined by the formula

$b= \begin{cases}\frac{1}{2} u_{1}^{2}+g \frac{\psi}{u_{1}}+\frac{g^{2}}{N_{1}^{2}}\left(1-e^{\frac{N_{1}^{2} \psi}{g u_{1}}}\right) & \left(-h_{1}<y<\eta(x)\right), \\ \frac{1}{2} u_{2}^{2}+g \frac{\psi}{u_{2}}+\frac{g^{2}}{N_{2}^{2}}\left(1-e^{\frac{N_{2}^{2} \psi}{g u_{2}}}\right) & \left(\eta(x)<y<h_{2}\right) .\end{cases}$

As a consequence, we can rewrite the DJL equation (11) as follows:

$\nabla^{2} \psi=\frac{N_{j}^{2}}{g u_{j}}\left\{g\left(y-\frac{\psi}{u_{j}}\right)+\frac{1}{2}\left(|\nabla \psi|^{2}-u_{j}^{2}\right)\right\}$,

where $j=1$ is related to the lower layer, and $j=2$ to the upper layer. Further, in accordance with relations (7) and (9), 
the continuity of pressure $p$ provides a non-linear boundary condition for stream function $\psi$ :

$\left[\rho(\psi)\left(|\nabla \psi|^{2}+2 g y-2 b(\psi)\right]=\left.0\right|_{y=\eta}\right.$.

Using the explicit form of functions $\rho(\psi)$ and $b(\psi)$, condition (13) can also be rewritten in detail as follows:

$$
\begin{aligned}
& 2 g\left(\rho_{1}-\rho_{2}\right) \eta= \\
& \quad=\left.\rho_{2}\left(|\nabla \psi|^{2}-u_{2}^{2}\right)\right|_{y=\eta(x)+0}-\left.\rho_{1}\left(|\nabla \psi|^{2}-u_{1}^{2}\right)\right|_{y=\eta(x)-0} .
\end{aligned}
$$

We reformulate this boundary condition in view of conservation of the total horizontal momentum in a steady two-layer flow, which has the integral formulation

$$
\int_{-h_{1}}^{h_{2}}\left(p+\rho u^{2}\right) \mathrm{d} y=C
$$

where constant $C$ is determined by the upstream condition. Excluding pressure $p$ from this relation using the Bernoulli equation (9) leads to the integral relation

$$
\rho_{1} \int_{-h_{1}}^{\eta(x)} e^{-\frac{N_{1}^{2} \psi}{g u_{1}}} \Psi_{1} \mathrm{~d} y+\rho_{2} \int_{\eta(x)}^{h_{2}} e^{-\frac{N_{2}^{2} \psi}{g u_{2}}} \Psi_{2} \mathrm{~d} y=C,
$$

where the integrand functions $\Psi_{j}$ are

$$
\Psi_{j}=\psi_{y}^{2}-\psi_{x}^{2}+u_{j}^{2}+2 g\left(\frac{\psi}{u_{j}}-y\right)-\frac{2 g^{2}}{N_{j}^{2}}\left(e^{\frac{N_{j}^{2} \psi}{g u_{j}}}-1\right),
$$

and constant $C$ depends on the parameters of the upstream flow as follows:

$$
\begin{aligned}
C=2 \rho_{1} g & {\left[\left(e^{\frac{N_{1}^{2} h_{1}}{g}}-1\right)\left(\frac{u_{1}^{2}}{N_{1}^{2}}+\frac{g^{2}}{N_{1}^{4}}\right)-\frac{g h_{1}}{N_{1}^{2}}\right]+} \\
+2 \rho_{2} g & {\left[\left(1-e^{-\frac{N_{2}^{2} h_{2}}{g}}\right)\left(\frac{u_{2}^{2}}{N_{2}^{2}}+\frac{g^{2}}{N_{2}^{4}}\right)-\frac{g h_{2}}{N_{2}^{2}}\right] . }
\end{aligned}
$$

It is important here that the integral relation (14) is equivalent to the boundary condition (13), which is rather simple. This equivalence can be checked immediately by differentiation of the relation (14) with respect to the variable $x$, so the integrals can be evaluated explicitly due to Eq. (12). Equation (14) will be used later instead of Eq. (13) by the construction model differential equation for the function $\eta(x)$ describing strongly non-linear waves.

\section{Non-dimensional formulation}

Now we introduce scaled independent variables $\bar{x}$ and $\bar{y}$ and scaled unknown functions $\bar{\eta}$ and $\bar{\psi}$ in order to reformulate the basic equations in the dimensionless form. That is, the fixed ratio $h_{1} / \pi$ is used as an appropriate length scale for $x$, $y$, and $\eta$, and normalized volume discharges $u_{j} h_{j} / \pi$ serve as the units for the stream function; thus, we have

$(x, y, \eta)=\frac{h_{1}}{\pi}(\bar{x}, \bar{y}, \bar{\eta}), \quad \psi=\frac{u_{j} h_{j}}{\pi} \bar{\psi}$

separately in the lower layer $(j=1)$ or in the upper layer $(j=2)$. The number $\pi$ is only introduced here due to the specific form of trigonometric modal functions which are typical for the exponential density (Eq. 8). The scaling procedure with this density profile uses the Boussinesq parameters $\sigma_{1}$ and $\sigma_{2}$ and the Atwood number $\mu$ defined by the formulae

$\sigma_{j}=\frac{N_{j}^{2} h_{j}}{\pi g} \quad(j=1,2), \quad \mu=\frac{\rho_{1}-\rho_{2}}{\rho_{2}}$.

Here, constants $\sigma_{j}$ characterize the slope of the density profile in continuously stratified layers, and parameter $\mu$ determines the density jump at the interface.

Following Turner (1973), we introduce the densimetric (or internal) Froude number

$$
F_{j}=\frac{u_{j}}{\sqrt{g_{j} h_{j}}} \quad(j=1,2),
$$

which presents scaled fluid velocity $u_{j}$ in the $j$ th layer, defined with reduced gravity acceleration $g_{j}=\left(\rho_{1}-\rho_{2}\right) g / \rho_{j}$. In addition to the Froude numbers $F_{j}$, it is also convenient to use the pair of Long's numbers $\lambda_{j}$ given by the formula

$\lambda_{j}=\frac{N_{j} h_{j}}{\pi u_{j}} \quad(j=1,2)$.

Long's numbers $\lambda_{j}$ are coupled with the Boussinesq parameters $\sigma_{1}$ and $\sigma_{2}$, the Atwood number $\mu$ and the Froude numbers $F_{j}$ by the relations

$\lambda_{1}^{2}=\frac{\pi \sigma_{1}(1+\mu)}{\mu F_{1}^{2}}, \quad \lambda_{2}^{2}=\frac{\pi \sigma_{2}}{\mu F_{2}^{2}}$.

Finally, we introduce the ratio of undisturbed thicknesses of the layers $r=h_{1} / h_{2}$. By that notation, we locate the bottom as $\bar{y}=-\pi$, and relation $\bar{y}=\pi / r$ defines the rigid lid. Thus, we obtain the equations for scaled stream function $\bar{\psi}$ and non-dimensional wave elevation $\bar{\eta}$ as follows (bar is omitted throughout what follows):

$\nabla^{2} \psi+\lambda_{1}^{2}(\psi-y)=\frac{1}{2} \sigma_{1}\left(|\nabla \psi|^{2}-1\right)$

in the lower layer $-\pi<y<\eta(x)$, and

$\nabla^{2} \psi+\lambda_{2}^{2} r^{2}(\psi-r y)=\frac{1}{2} \sigma_{2}\left(|\nabla \psi|^{2}-r^{2}\right)$

in the upper layer $\eta(x)<y<\pi / r$. Kinematic boundary conditions (10) can be rewritten now as follows:

$\psi(x,-\pi)=-\pi, \quad \psi(x, \eta(x))=0, \quad \psi(x, \pi / r)=\pi$. 
Correspondingly, Eq. (13), providing continuity of pressure at interface $y=\eta(x)$, leads to the non-linear boundary condition

$2 \eta=\left.F_{2}^{2}\left(|\nabla \psi|^{2}-r^{2}\right)\right|_{y=\eta+0}-\left.F_{1}^{2}\left(|\nabla \psi|^{2}-1\right)\right|_{y=\eta-0}$,

and the dimensionless version of integral relation (14) takes the form

$\int_{-\pi}^{\eta} e^{-\sigma_{1} \psi} \Psi_{1} \mathrm{~d} y+\int_{\eta}^{\pi / r} e^{-\sigma_{2} \psi} \Psi_{2} \mathrm{~d} y=C$

where the following is denoted:

$\Psi_{1}=\frac{\mu F_{1}^{2}}{2}\left(\psi_{y}^{2}-\psi_{x}^{2}+1\right)+\frac{1+\mu}{\pi}\left(\psi-y-\frac{e^{\sigma_{1} \psi}-1}{\sigma_{1}}\right)$

$\Psi_{2}=\frac{\mu F_{2}^{2}}{2 r^{3}}\left(\psi_{y}^{2}-\psi_{x}^{2}+r^{2}\right)+\frac{1}{\pi r}\left(\psi-r y-\frac{e^{\sigma_{2} \psi}-1}{\sigma_{2}}\right)$.

Constant

$$
\begin{aligned}
C & =\pi \mu\left(F_{1}^{2}+\frac{F_{2}^{2}}{r^{2}}\right)+ \\
& +(1+\mu) \frac{e^{\sigma_{1} \pi}-1-\sigma_{1} \pi}{\pi\left(\lambda_{1}^{2}+\sigma_{1}^{2}\right)}+\frac{1-\sigma_{2} \pi-e^{-\sigma_{2} \pi}}{\pi r^{2}\left(\lambda_{2}^{2}+\sigma_{2}^{2}\right)}
\end{aligned}
$$

is chosen here so that the horizontal upstream flow given by the solution

$\eta=0, \quad \psi_{0}(y)= \begin{cases}y & (-\pi<y<0), \\ r y & (0<y<\pi / r)\end{cases}$

satisfies momentum relation (21).

The model of fully non-linear travelling waves in a twolayer irrotational flow, with the interface $y=\eta(x)$ between the fluids with constant densities $\rho_{2}$ in the upper layer and $\rho_{1}>\rho_{2}$ in the lower layer, can be specified as follows. In this limit case, at least formally, the Boussinesq parameters $\sigma_{j}$ and Long's numbers $\lambda_{j}$ vanish: $\sigma_{1}=\sigma_{2}=\lambda_{1}=\lambda_{2}=0$. Therefore, we obtain the Laplace equation

$\nabla^{2} \psi=0$

instead of Eqs. (17)-(18), but all the boundary conditions (19) and (20) still remain unchanged.

\section{Spectrum of harmonic waves}

In many cases, a parametric range of solitary waves can be determined a priori as the domain being supercritical with respect to the spectrum of small-amplitude sinusoidal waves. It is helpful while the critical phase speed can be simply defined from the dispersion relation of infinitesimal waves. In our case, linearizing of Eqs. (17)-(20) for the upstream solution (22) leads to the dispersion relation

$\Delta\left(k ; F_{1}, F_{2}\right)=0$

for stationary harmonic wave packets

$\eta(x)=a e^{i k x}, \quad \psi=\psi_{0}(y)+W(y) e^{i k x}$.

Here, $k$ is the non-dimensional wave number, $a$ is the amplitude of the interfacial wave, and $W(y)$ is the modal eigenfunction which describes deformation of streamlines within the fluid layers. For the given Long numbers $\lambda_{1}$ and $\lambda_{2}$ and the Boussinesq parameters $\sigma_{1}$ and $\sigma_{2}$, we also introduce nondimensional values

$\varkappa_{j}=\sqrt{\left|\lambda_{j}^{2}-k_{j}^{2}-\frac{1}{4} \pi^{2} \sigma_{j}^{2}\right|} \quad(j=1,2)$,

where $k_{1}=r k$ and $k_{2}=k$ are dimensionless wave numbers specified for each layer. According to these notations, dispersion function $\Delta\left(k ; F_{1}, F_{2}\right)$ in $(24)$ has the form

$\Delta=F_{1}^{2}\left(\varkappa_{1} \operatorname{Cot}_{1} \varkappa_{1}+\frac{\pi \sigma_{1}}{2}\right)+F_{2}^{2}\left(\varkappa_{2} \operatorname{Cot}_{2} \varkappa_{2}-\frac{\pi \sigma_{2}}{2}\right)-1$,

where functions $\operatorname{Cot}_{j}(j=1,2)$ are denoted as follows:

$\operatorname{Cot}_{j} \varkappa_{j}= \begin{cases}\operatorname{Cot} \varkappa_{j} & \left(\lambda_{j}^{2}>k_{j}^{2}+\frac{1}{4} \pi^{2} \sigma_{j}^{2}\right) \\ \operatorname{coth} \varkappa_{j} & \left(\lambda_{j}^{2}<k_{j}^{2}+\frac{1}{4} \pi^{2} \sigma_{j}^{2}\right) .\end{cases}$

In fact, function $\Delta$ takes such a combined form since modal function $W(y)$ depends on $y$ trigonometrically or hyperbolically, if the radicand term $\lambda_{j}^{2}-k_{j}^{2}-\frac{1}{4} \pi^{2} \sigma_{j}^{2}$ in (25) is positive or negative. Explicit formulae for these modal eigenfunctions $W(y)$ are given in Appendix A.

A spectrum of stationary harmonic waves, defined on the $\left(F_{1}, F_{2}\right)$ plane, is formed by the Froude points $\left(F_{1}, F_{2}\right)$ so that dispersion function $\Delta\left(k ; F_{1}, F_{2}\right)$, which is even in $k$, has at least one pair of real roots $\pm k$. Wave modes differ by the number of these pairs, and this number can change only by passing of the root across the value $k=0$. Therefore, the modal bounds should satisfy the equation $\Delta\left(0 ; F_{1}, F_{2}\right)=0$; these bounds are defined by separate branches of the curve

$$
\begin{aligned}
& F_{1}^{2}\left\{\sqrt{\lambda_{1}^{2}-\left(\frac{\pi \sigma_{1}}{2}\right)^{2}} \operatorname{Cot} \sqrt{\lambda_{1}^{2}-\left(\frac{\pi \sigma_{1}}{2}\right)^{2}}-\frac{\pi \sigma_{1}}{2}\right\}+ \\
& +F_{2}^{2}\left\{\sqrt{\lambda_{2}^{2}-\left(\frac{\pi \sigma_{2}}{2}\right)^{2}} \operatorname{Cot} \sqrt{\lambda_{2}^{2}-\left(\frac{\pi \sigma_{2}}{2}\right)^{2}}+\frac{\pi \sigma_{2}}{2}\right\}=1,
\end{aligned}
$$

where parameters $\lambda_{j}$ should be coupled with the Froude numbers $F_{j}$ using the formula (16).

We emphasize that parameters $\sigma_{j}$ characterize the slope of the density profile in continuously stratified layers, and $\mu$ defines the density jump at the interface. As usual, all these 


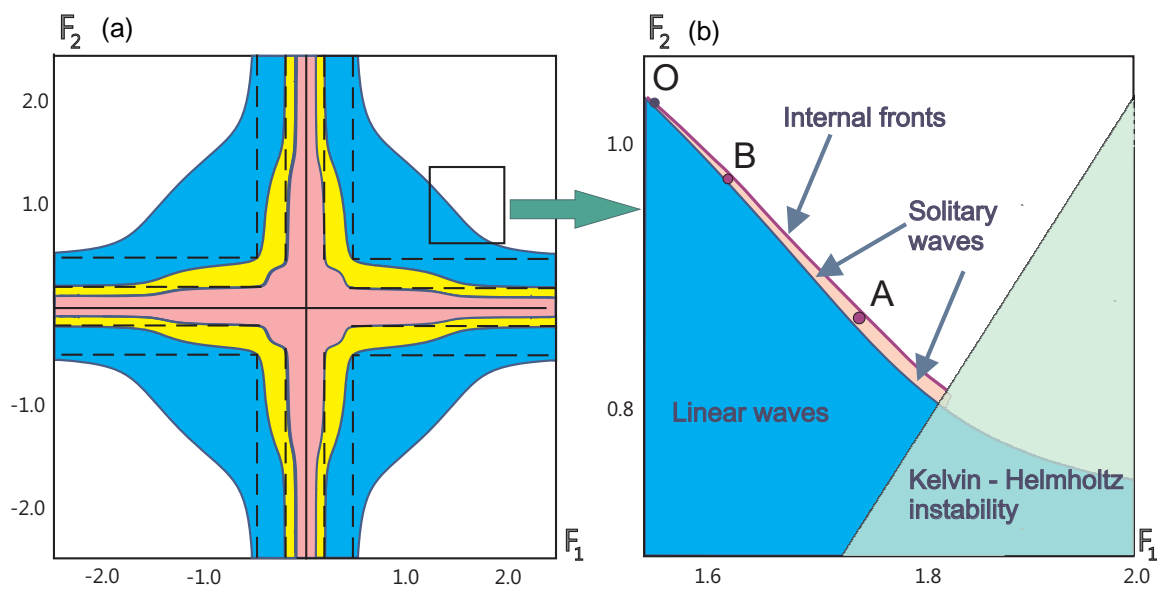

Figure 2. Spectrum of linear waves (coloured modes 1-3) (a) and fragment of the parametric domain of solitary waves (b).

parameters are small in the case of low stratification. However, the interfacial mode dominates over the modes of internal waves in stratified layers when $\sigma_{j} \ll \mu$ is valid. In this limit case, linearized boundary conditions (19)-(20), considered together with the linear Laplace equation (23), lead to the standard dispersion relation of two-layer fluid:

$F_{1}^{2} r k \operatorname{coth} r k+F_{2}^{2} k \operatorname{coth} k=1$.

This relation determines only a single pair of real wave numbers of the interfacial mode, so the spectral domain of a perfect two-layer system occupies the unit disk:

$F_{1}^{2}+F_{2}^{2} \leqslant 1$.

The 2.5-layer model starts with the hypotheses that the Boussinesq parameters $\sigma_{1}$ and $\sigma_{2}$ and the Atwood number $\mu$ are of the same order, so we can use a single small parameter $\sigma$ by setting

$\sigma=\sigma_{1}=\sigma_{2}=\mu$.

The limit passage $\sigma \rightarrow 0$ is singular because Long's numbers $\lambda_{j}$ involve the ratios $\sigma_{j} / \mu$ in formula (16). However, condition (29) allows us to simplify the spectral portrait; hence, modal curve (26) defining the critical wave speeds takes the form

$\sqrt{\pi} F_{1} \operatorname{Cot} \frac{\sqrt{\pi}}{F_{1}}+\sqrt{\pi} F_{2} \operatorname{Cot} \frac{\sqrt{\pi}}{F_{2}}=1$.

Figure 2 demonstrates the parts of the spectrum defined by curve (30) for the dominating modes. The domain covered only by the first mode is marked with the blue colour. Correspondingly, the embedded domain of the second mode is highlighted with the yellow colour, and the third mode is marked with the pink colour. It is important that this spectrum differs essentially from the ordinary two-layer spectrum (28), even when the flow is characterized by a pair of the
Froude numbers $F_{1}$ and $F_{2}$ defined in the same manner. We especially note that the 2.5-layer spectrum extends infinitely on the spectral plane by involving unbounded Froude numbers $F_{j}$.

\section{The non-linear long-wave model}

The derivation procedure of the non-linear long-wave 2.5layer model should involve, in accordance with hypothesis (29), the slow horizontal variable $\xi=\sqrt{\sigma} x$, as was demonstrated by Benney and Ko (1978) in the case of slight linear stratification. Scaling with the parameter $\sigma$ gives the equation

$\sigma \psi_{\xi \xi}+\psi_{y y}+\lambda_{1}^{2}(\psi-y)=\frac{1}{2} \sigma\left(\sigma \psi_{\xi}^{2}+\psi_{y}^{2}-1\right)$

in the lower layer $-\pi<y<\eta(\xi)$, and

$\sigma \psi_{\xi \xi}+\psi_{y y}+\lambda_{2}^{2} r^{2}(\psi-r y)=\frac{1}{2} \sigma\left(\sigma \psi_{\xi}^{2}+\psi_{y}^{2}-r^{2}\right)$

in the upper layer $\eta(\xi)<y<\pi / r$. Kinematic boundary conditions (10) can be rewritten now as follows:

$\psi(\xi,-\pi)=-\pi, \quad \psi(\xi, \eta(\xi))=0, \quad \psi(\xi, \pi / r)=\pi$.

We find that stream function $\psi$ is expanded in a power series with respect to $\sigma$ as

$\psi=\psi^{(0)}(\xi, y)+\sigma \psi^{(1)}(\xi, y)+\ldots$,

where the leading-order term $\psi^{(0)}$ defines the hydrostatic mode, and the coefficient $\psi^{(1)}$ provides the correction due to non-linear dispersion. All these coefficients $\psi^{(k)}$ can be uniquely determined from Eqs. (31) and (32) (with fixed Long numbers $\lambda_{1}$ and $\lambda_{2}$ ) under kinematic boundary condition (33). Thus, we obtain

$\psi^{(0)}=y-\eta \frac{\sin \alpha_{1}(y)}{\sin \alpha_{1}(\eta)} \quad(-\pi<y<\eta)$ 
and

$\psi^{(0)}=r y-r \eta \frac{\sin \alpha_{2}(y)}{\sin \alpha_{2}(\eta)} \quad(\eta<y<\pi / r)$,

where

$\alpha_{1}(y)=\lambda_{1}(\pi+y), \quad \alpha_{2}(y)=\lambda_{2}(\pi-r y)$.

The final form of the dispersive term $\psi^{(1)}$ is much more complicated; therefore, this coefficient is given in Appendix A.

Now we substitute power expansion (34) for function $\psi$ into the scaled version of integral relation (21) and truncate the terms with the powers higher than the first power of $\sigma$. By that, Eq. (21) reduces to the first-order ordinary differential equation for the wave elevation $\eta(x)$ and is written as

$\left(\frac{\mathrm{d} \eta}{\mathrm{d} x}\right)^{2}=\eta^{2} \frac{D\left(\eta ; F_{1}, F_{2}\right)}{Q\left(\eta ; F_{1}, F_{2}\right)}$.

Here function $D$ is given by the formula

$$
\begin{aligned}
& D \\
& \left.\quad \eta ; F_{1}, F_{2}\right)= \\
& \quad=\sqrt{\pi} F_{1} \cot \alpha_{1}(\eta)+\sqrt{\pi} F_{2} \cot \alpha_{2}(\eta)+\frac{1}{3}(1-r) \eta-1,
\end{aligned}
$$

where $\alpha_{1}$ and $\alpha_{2}$ should be taken as

$\alpha_{1}(\eta)=\frac{\pi+\eta}{\sqrt{\pi} F_{1}}, \quad \alpha_{2}(\eta)=\frac{\pi-r \eta}{\sqrt{\pi} F_{2}}$,

since we have at the leading order in $\sigma$ the relations $\lambda_{j}=$ $1 / \sqrt{\pi} F_{j}(j=1,2)$ obtained under condition (29). Denominator $Q$ in Eq. (36) has a complicated form; therefore, this function is given in Appendix C. Solitary-wave solutions of Eq. (36) are given in implicit form by the formula

$$
x= \pm \int_{a}^{\eta} \sqrt{\frac{Q\left(s ; F_{1}, F_{2}\right)}{D\left(s ; F_{1}, F_{2}\right)}} \frac{\mathrm{d} s}{s},
$$

where parameter $a$ determines the non-dimensional amplitude of the wave.

Small-amplitude waves can be modelled by a simplified weakly non-linear version of Eq. (36) with the form

$$
\left(\frac{\mathrm{d} \eta}{\mathrm{d} x}\right)^{2}=\eta^{2} \frac{D_{0}+D_{1} \eta+D_{2} \eta^{2}}{Q\left(0 ; F_{1}, F_{2}\right)}
$$

where the coefficients $D_{0}=D\left(0 ; F_{1}, F_{2}\right) \quad$ and $D_{1}=D_{\eta}^{\prime}\left(0 ; F_{1}, F_{2}\right)$ are

$D_{0}=\sqrt{\pi} F_{1} \cot \frac{\sqrt{\pi}}{F_{1}}+\sqrt{\pi} F_{2} \cot \frac{\sqrt{\pi}}{F_{2}}-1$,

$D_{1}=-\cot ^{2} \frac{\sqrt{\pi}}{F_{1}}+r \cot ^{2} \frac{\sqrt{\pi}}{F_{2}}+\frac{2}{3}(r-1)$,

and the explicit form of coefficient $D_{2}$ is not important here. This model takes into account the balance of quadratic and cubic non-linearities in the weakly non-linear KdV-mKdVGardner model (Kakutani and Yamasaki, 1978; Gear and Grimshaw, 1983; Helfrich and Melville, 2006; Grimshaw et al., 2002).

Solitary-wave regimes are obtained depending on the multiplicity of the roots $a_{j}\left(F_{1}, F_{2}, r\right)(j=1,2)$ of the numerator on the right-hand side of Eq. (39). The profile of a solitary wave is given by the formulae

$\eta(x)=a \frac{1-\tanh ^{2} k x}{1-\theta^{2} \tanh ^{2} k x}, \quad k=\frac{a \sqrt{3 / q_{*}}}{2 \theta}$,

with $q_{*}=Q(0), a=a_{1}$ and $\theta^{2}=a_{1} / a_{2}<1$, and the bore (internal front) corresponds to the double root $a=a_{1}=a_{2}$; it has the following profile:

$\eta(x)=\frac{a}{2}(1+\tanh k x), \quad k=\frac{a \sqrt{3 / q_{*}}}{2}$.

The parametric range of strongly non-linear solitary waves described by Eq. (36) is formed by the domain in the $\left(F_{1}, F_{2}\right)$ plane where the radical function $Q / D$ in (38) is ensured to be non-negative. It is easy to check that $Q\left(0 ; F_{1}, F_{2}\right)>0$; hence, function $Q\left(s ; F_{1}, F_{2}\right)$ is positive in the vicinity of point $s=0$. Therefore, function $D$ plays the determining role here. Depending on $F_{1}$ and $F_{2}$, this function can change sign even by small $s$, where the leading-order coefficient $D_{0}$ from formula (39) dominates. As a consequence, the map of solitary-wave regimes is formed by the Froude numbers $\left(F_{1}, F_{2}\right)$ such that inequality $D_{0}\left(F_{1}, F_{2}\right)>0$ holds. Indeed, this inequality defines the range of non-linear waves, which are supercritical with respect to the phase speed of linear harmonic wave packets (see Fig. 2).

\section{Marginally stable layered flows}

Large-amplitude internal waves are generated in deep ocean layers due to the interaction of internal tides with irregular bottom topography near underwater ridges (Morozov, 1995, 2018; Morozov et al., 2010). These waves play a significant role in the energy transformation and mass transport in the oceanic stratified flows, while they intensify mixing of the abyssal waters. Note that internal Froude numbers $F_{1}$ and $F_{2}$ characterize the magnitude of the velocity jump at the interface in upstream flow. The shear $u_{1} \neq u_{2}$ between the layers can initiate the development of the Kelvin-Helmholtz instability which provides non-stationary formation of billow trains (Thorpe, 1985; Drazin, 2002). In this context, longwave perturbations give the greatest contribution to this instability due to their increased power intensity. Constant twolayer flow is linearly stable in the long-wave limit if the inequality

$\left|u_{1}-u_{2}\right|<\sqrt{\frac{g\left(\rho_{1}-\rho_{2}\right)\left(\rho_{1} h_{2}+\rho_{2} h_{1}\right)}{\rho_{1} \rho_{2}}}$ 
holds, and this flow is unstable in the opposite case (Lamb, 1932). Exactly the same bound (40) follows from the nonlinear stability criteria predicted by the shallow water theory (Ovsyannikov, 1979; see also Baines, 1995; Gavrilyuk et al., 2017) for a variable difference $\left|\bar{u}_{1}-\bar{u}_{2}\right|$ and variable layer thicknesses

$\bar{h}_{1}(x, t)=h_{1}+\eta(x, t), \quad \bar{h}_{2}(x, t)=h_{2}-\eta(x, t)$.

In this case, velocity $\bar{u}_{j}$ is the horizontal fluid velocity averaged over the depth of the $j$ th layer $(j=1,2)$,

$$
\begin{aligned}
& \bar{u}_{1}(x, t)=\frac{1}{\bar{h}_{1}(x, t)} \int_{-h_{1}}^{\eta(x, t)} u(x, y, t) \mathrm{d} y, \\
& \bar{u}_{2}(x, t)=\frac{1}{\bar{h}_{2}(x, t)} \int_{\eta(x, t)}^{h_{2}} u(x, y, t) \mathrm{d} y .
\end{aligned}
$$

Condition (40) considered for non-constant velocities $\bar{u}_{1}$ and $\bar{u}_{2}$ and non-constant layer thicknesses $\bar{h}_{1}$ and $\bar{h}_{2}\left(\bar{h}_{1}+\bar{h}_{2}=\right.$ $h_{1}+h_{2}=$ const) provides hyperbolicity of hydrostatic twolayer shallow water equations

$\bar{h}_{1 t}+\left(\bar{u}_{1} \bar{h}_{1}\right)_{x}=0, \quad \bar{h}_{2 t}+\left(\bar{u}_{2} \bar{h}_{2}\right)_{x}=0$,

$\rho_{1}\left(\bar{u}_{1 t}+\bar{u}_{1} \bar{u}_{1 x}+g \bar{h}_{1 x}\right)=\rho_{2}\left(\bar{u}_{2 t}+\bar{u}_{2} \bar{u}_{2 x}+g \bar{h}_{1 x}\right)$.

In accordance with this non-linear formulation, layered flow can be locally unstable due to increased wave-induced shear even when the constant upstream flow satisfies condition (40). This marginal instability of internal solitary waves was observed in laboratory experiments (Gavrilov, 1994; Grue et al., 1999, 2000; Carr et al., 2008; Fructus et al., 2009) and studied theoretically (Choi et al., 2009; Almgren et al., 2012). Mathematical models of layered stable-unstable flows, which account also for the non-linear dispersion of the waves and non-hydrostatic effects, are currently under intense discussion in Barros and Choi (2013), Lannes and Ming (2015), Duchene et al. (2016), Liapidevskii et al. (2018), and Makarenko et al. (2018). However, the hydrostatic condition (40) seems to be appropriate by estimation of the stability limit with non-dimensional parameters from Sect. 3. Hence, we obtain the stability domain

$\left|\sqrt{r} F_{1}-F_{2}\right|<\sqrt{1+r}$,

which gives an admissible set of the Froude points $\left(F_{1}, F_{2}\right)$ defined for the instant ratio of local layer depths $r=\bar{h}_{1} / \bar{h}_{2}$ and local Froude numbers in the solitary-wave-type flow described by stationary solution (38). This domain is shown for $r=3$ in Fig. 2 (right panel) as the unshadowed domain in the selected part of quarter-plane $\left(F_{1}, F_{2}\right)$.

\section{Waves in abyssal shear flows}

We present in this section a comparison of solutions of suggested mathematical models with the field data measured

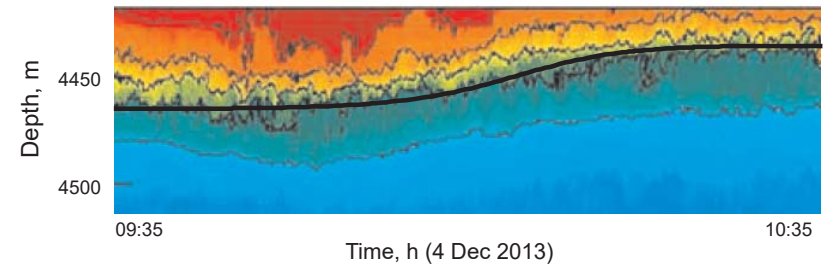

Figure 3. Internal front in abyssal stratified flow.

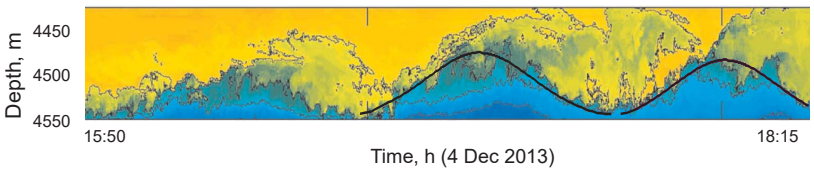

Figure 4. Train of interfacial solitary waves affected by the KelvinHelmholtz instability.

for internal solitary waves in weakly stratified abyssal currents. Figures 3 and 4 demonstrate fragments of temperature distribution in a quasi-steady shear bottom flow recorded from a mooring station with a $350 \mathrm{~m}$ line of thermistors located over a depth of $4720 \mathrm{~m}$ at the entrance to the Romanche Fracture Zone in the equatorial Atlantic (Van Haren et al., 2014). Trains of short-period (20/30 min) internal waves modulated by tides propagate here along a sharp interface corresponding to the $0.85^{\circ} \mathrm{C}$ isotherm which separates the lower layer of cold Antarctic Bottom Water (AABW) from the overlying warmer $\mathrm{AABW}$ layer with temperature $\theta<2.0^{\circ} \mathrm{C}$. It is seen from the figures that moored temperature data show permanently marginal stability of the flow with the Richardson numbers $0.25<R i<1$. Tidal amplification of the shear triggers the formation of small-scale overturns which create long trains of the Kelvin-Helmholtz billows. Bold curves in Figs. 3 and 4 show overlapped profiles of internal waves calculated from Eq. (38). Figure 3 demonstrates a front-like wave (internal bore) affected by moderate small-scale shear instability. The non-dimensional amplitude of this wave is $a=\eta_{\max } / h_{1}=0.16$, and the calculated solution corresponds to Froude point A with coordinates $\left(F_{1}, F_{2}\right)=(1.719,0.891)$ shown in Fig. 2 (panel $\left.(\mathbf{b})\right)$. Point A belongs to the bore diagram which is tangential to the spectrum boundary at point $\mathrm{O}$ corresponding to the vanishing amplitude limit $a=0$. Figure 4 demonstrates the train of three subsequent waves with intense overturns distributed along with gently sloping wave tops. The amplitude of the leading wave shown in Fig. 4 is clearly lower than the amplitudes of the following two waves, which occur randomly and are almost identical. The solitary-wave type of these two waves corresponds to Froude point B with coordinates $\left(F_{1}, F_{2}\right)=(1.634,0.959)$.

The upstream parameters used in the calculation were chosen from conductivity, temperature and pressure (CTD) and lowered acoustic Doppler current profiler (LADCP) data of 


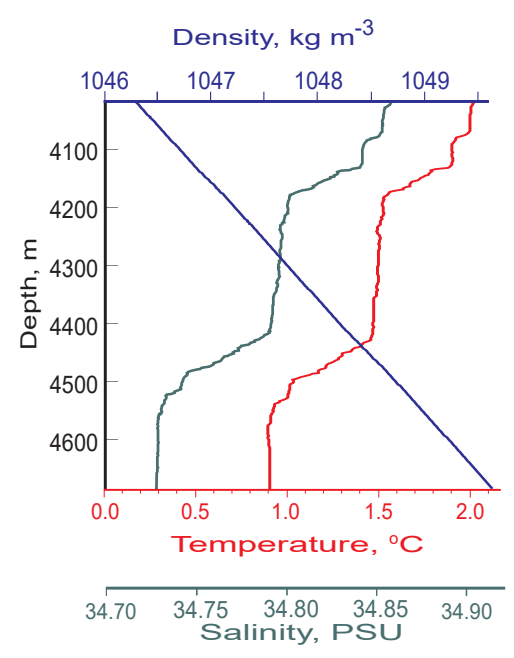

Figure 5. Profiles of the density, salinity and temperature.

density and currents measured immediately at the fronts of selected waves. Undisturbed depth of lower-layer $h_{1}$ was fixed by the equilibrium level of isotherm $0.85^{\circ} \mathrm{C}$ recorded at the buoy station shortly before the arrival of the large wave. In fact, this level changed gradually due to the tide, so that the value of $h_{1}$ varied within a day in the range from 170 to $240 \mathrm{~m}$. The depth of the upper layer $h_{2}$ was evaluated by fixing the appropriate overlying isotherm, which remained almost horizontal; hence, this depth can be considered the "rigid lid" in the model of the 2.5-layer flow. This kind of "rigid" streamline appears frequently in abyssal shear flows due to the formation of critical layers which separate clearly the currents with different thermohaline parameters (Morozov et al., 2012). The estimated depth $h_{2}$ also depends on the tidal activity, and this value varied in a wider range from 70 to $350 \mathrm{~m}$. Nevertheless, the resulting total depth $h=h_{1}+h_{2}$ did not exceed the thickness of the entire AABW layer confined below a depth of $4000 \mathrm{~m}$.

Upstream velocities $u_{1}$ and $u_{2}$ were assumed as $u_{1}=$ $35 / 45 \mathrm{~cm} \mathrm{~s}^{-1}$ and $u_{2}=10 / 20 \mathrm{~cm} \mathrm{~s}^{-1}$. These values correspond to the mean velocities measured in the western part of the Romanche Fracture Zone by a velocity LADCP profiler in the cruises of the R/V Akademik Sergey Vavilov
(Tarakanov et al., 2013) and measured on the mooring station (Van Haren et al., 2014). Maximal velocity of the flow in the lower layer, recorded by the mooring station within 6 months, is as high as $65 \mathrm{~cm} \mathrm{~s}^{-1}$. We consider that intense shear is induced here by the permanent inflow of bottom waters to the fracture zone through a narrow gap in its southern wall. Figure 5 demonstrates vertical profiles of temperature, salinity, and density measured on the shipborne mooring over a depth of $4760 \mathrm{~m}$ downstream from the location of the bottom station. These thermohaline data highlight the layering of near-bottom flow with very smooth pycnoclines thickened due to intense mixing. In addition, the slope of an almost linear density profile shown in Fig. 5 permits us to evaluate the Boussinesq parameter $\sigma$ by the value $\sigma=0.0027$. The Froude points $\left(F_{1}, F_{2}\right)$ related to these parameters match adequately with the range of internal solitary waves shown in the right panel of Fig. 2 (narrow band marked by the orange colour). The narrowness of this range reveals anomalously low-amplitude dispersion of solitary waves, which agrees with the field observations.

\section{Conclusions}

In this paper we have considered the problem of internal stationary waves at the interface between exponentially stratified fluid layers. We demonstrated that the non-linear DJL model of weakly stratified 2.5-layer fluid flow can be reduced explicitly to an approximate non-linear ordinary differential equation describing large-amplitude internal solitary waves. The parametric range of solitary waves is described, including regimes of broad plateau-shaped solitary waves and internal fronts. These wave regimes can be affected by the Kelvin-Helmholtz instability induced by the velocity shear at the interface; hence, the marginal stability of internal waves could explain the formation mechanism of very long billow trains observed in the Romanche Fracture Zone.

Data availability. The data can be requested from author Eugene Morozov by the e-mail egmorozov@ mail.ru. 


\section{Appendix A: Modal functions of the linearized problem}

Eigenfunction $W(y)$ considered in the strip $-\pi<y<0$ has the form

$$
W=a_{1} e^{\frac{1}{2} \sigma_{1} y} \begin{cases}e^{\varkappa_{1} \pi} \frac{\sinh \varkappa_{1}(\pi+y)}{\varkappa_{1}} & \left(\lambda_{1}^{2}<k_{1}^{2}+\frac{\pi^{2}}{4} \sigma_{1}^{2}\right) \\ \pi+y & \left(\lambda_{1}^{2}=k_{1}^{2}+\frac{\pi^{2}}{4} \sigma_{1}^{2}\right) \\ \frac{\sin \varkappa_{1}(\pi+y)}{\varkappa_{1}} & \left(\lambda_{1}^{2}>k_{1}^{2}+\frac{\pi^{2}}{4} \sigma_{1}^{2}\right) .\end{cases}
$$

Similarly, eigenfunction $W(y)$ defined in the upper layer which corresponds to the strip $0<y<\pi / r$ has the form

$$
W=a_{2} e^{\frac{1}{2} \sigma_{2} y} \begin{cases}e^{\varkappa_{2} \pi} \frac{\sinh \varkappa_{2}(\pi-r y)}{\varkappa_{2}} & \left(\lambda_{2}^{2}<k_{2}^{2}+\frac{\pi^{2}}{4} \sigma_{2}^{2}\right) \\ \pi-r y & \left(\lambda_{2}^{2}=k_{2}^{2}+\frac{\pi^{2}}{4} \sigma_{2}^{2}\right) \\ \frac{\sin s_{1}(\pi-r y)}{\varkappa_{2}} & \left(\lambda_{2}^{2}>k_{2}^{2}+\frac{\pi^{2}}{4} \sigma_{2}^{2}\right) .\end{cases}
$$

Appendix C: Denominator of the non-linear long-wave equation

Denominator $Q$ in Eq. (36) has the form

$$
\begin{aligned}
& 2 Q\left(\eta ; F_{1}, F_{2}\right)= \\
& \quad\left(\pi F_{1}^{2}-2 \sqrt{\pi} F_{1} \eta \cot \alpha_{1}(\eta)+\eta^{2} \cot ^{2} \alpha_{1}(\eta)\right) \times \\
& \quad \times\left(\frac{\eta+\pi}{\sin ^{2} \alpha_{1}(\eta)}-\sqrt{\pi} F_{1} \cot \alpha_{1}(\eta)\right)+ \\
& \quad+\left(\frac{\pi F_{2}^{2}}{r^{2}}-2 \frac{\sqrt{\pi} F_{2}}{r} \eta \cot \alpha_{2}(\eta)+\eta^{2} \cot ^{2} \alpha_{2}(\eta)\right) \times \\
& \quad \times\left(\frac{\pi-r \eta}{\sin ^{2} \alpha_{2}(\eta)}-\sqrt{\pi} F_{2} \cot \alpha_{2}(\eta)\right),
\end{aligned}
$$

where functions $\alpha_{j}(\eta)$ are given by formula (37), which is an approximate version of Eq. (35).
The dimensionless wave numbers $\varkappa_{j}(j=1,2)$ are introduced in formula (25), and the factors $a_{j}$ are the amplitude parameters.

\section{Appendix B: Dispersive term of the long-wave expansion}

The coefficient $\psi^{(1)}(\xi, y)$ which gives the correction due to the dispersion in the power expansion (34) has the form

$$
\begin{aligned}
& \psi^{(1)}=\frac{\eta(\eta-y)}{2} \frac{\sin \alpha_{1}(y)}{\sin \alpha_{1}(\eta)}+\frac{\sin \alpha_{1}(y)}{2 \lambda_{1}}\left(\frac{\eta}{\sin \alpha_{1}(\eta)}\right)_{\xi \xi} \times \\
& \times\left\{(\pi+\eta) \cot \alpha_{1}(\eta)-(\pi+y) \cot \alpha_{1}(y)\right\}+\frac{\eta^{2}}{6} \times \\
& \times\left\{\frac{\sin \lambda_{1}(y-\eta)-\sin \alpha_{1}(y)}{\sin ^{3} \alpha_{1}(\eta)}+\frac{1+\sin ^{2} \alpha_{1}(y)}{\sin ^{2} \alpha_{1}(\eta)}-\frac{\sin \alpha_{1}(y)}{\sin \alpha_{1}(\eta)}\right\}
\end{aligned}
$$

in the lower layer $-\pi<y<\eta$. Similarly, we have

$$
\begin{aligned}
& \psi^{(1)}=\frac{r^{2} \eta(\eta-y)}{2} \frac{\sin \alpha_{2}(y)}{\sin \alpha_{2}(\eta)}+\frac{\sin \alpha_{2}(y)}{2 \lambda_{2}}\left(\frac{\eta}{\sin \alpha_{2}(\eta)}\right)_{\xi \xi} \times \\
& \times\left\{(y-\pi / r) \cot \alpha_{2}(y)-(\eta-\pi / r) \cot \alpha_{2}(\eta)\right\}+\frac{r^{2} \eta^{2}}{6} \times \\
& \times\left\{\frac{\sin \lambda_{2} r(\eta-y)-\sin \alpha_{2}(y)}{\sin ^{3} \alpha_{2}(\eta)}+\frac{1+\sin ^{2} \alpha_{2}(y)}{\sin ^{2} \alpha_{2}(\eta)}-\frac{\sin \alpha_{2}(y)}{\sin \alpha_{2}(\eta)}\right\}
\end{aligned}
$$

in the upper layer $\eta<y<\pi / r$. Here the functions $\alpha_{j}$ are given by the formula (35). 
Author contributions. All authors made the same contribution to this work.

Competing interests. The authors declare that they have no conflict of interest.

Special issue statement. This article is part of the special issue "Extreme internal wave events". It is a result of the EGU, Vienna, Austria, 23-28 April 2017.

Acknowledgements. This work was supported by the Russian Foundation for Basic Research (grant nos. 15-01-03942, 17-0800085 and 18-01-00648) and Interdisciplinary Program II.1 of SB RAS (project no. 2). Roman Tarakanov was supported by the Russian Science Foundation (grant no. 16-17-10149).

Edited by: Kateryna Terletska

Reviewed by: two anonymous referees

\section{References}

Almgren, A., Camassa, R., and Tiron, R.: Shear instability of internal solitary waves in Euler fluids with thin pycnoclines, J. Fluid Mech., 710, 324-361, 2012.

Baines, P. G.: Topographic effects in stratified flows, Cambridge University Press, Cambridge, UK, 1995.

Barros, R. and Choi, W.: On regularizing the strongly nonlinear model for two-dimensional internal waves, Physica D, 264, 2734, 2013.

Benney, D. J. and Ko, D. R. S.: The propagation of long large amplitude internal waves, Stud. Appl. Math., 59, 187-199, 1978.

Camassa, R. and Tiron, R.: Optimal two-layer approximation for continuous density stratification, J. Fluid Mech., 669, 32-54, 2011.

Carr, M., Fructus, D., Grue, J., Jensen, A., and Davies, P. A.: Convectively induced shear instability in large amplitude internal solitary waves, Phys. Fluids, 20, 12660, https://doi.org/10.1063/1.3030947, 2008.

Choi, W. and Camassa, R.: Fully nonlinear internal waves in a twofluid system, J. Fluid Mech., 396, 1-36, 1999.

Choi, W., Barros, R., and Jo, T.-C.: A regularized model for strongly nonlinear internal solitary waves, J. Fluid Mech., 629, 73-85, 2009.

Drazin, P.: Introduction to hydrodynamic stability, Cambridge Univ. Press, Cambridge UK, 2002.

Duchene, V., Israwi, S., and Talhouk, R.: A new class of two-layer Green - Naghdi systems with improved frequency dispersion, Stud. Appl. Math., 137, 356-415, 2016.

Fructus, D., Carr, M., Grue, J., Jensen, A., and Davies, P.: Shearinduced breaking of large internal solitary waves, J. Fluid Mech., 620, 1-29, 2009.

Gavrilov, N. V.: Internal solitary waves and smooth bores which are stationary in a laboratory coordinate system, J. Appl. Mech. Techn. Phys., 35, 29-33, 1994.
Gavrilyuk, S. L., Makarenko, N. I., and Sukhinin, S. V.: Waves in continuous media, Lecture notes in geosystem mathematics and computing, Birkhäuser/Springer, Cham, Switzerland, 2017.

Gear, J. A. and Grimshaw, R.: A second-order theory for solitary waves in shallow fluids, Phys. Fluids, 26, 14-29, 1983.

Grimshaw, R., Pelinovsky, E., and Poloukhina, O.: Higher-order Korteweg-de Vries models for internal solitary waves in a stratified shear flow with a free surface, Nonlin. Processes Geophys., 9, 221-235, https://doi.org/10.5194/npg-9-221-2002, 2002.

Grue, J., Jensen, A., Rusås, P.-O., and Sveen, J. K.: Properties of large-amplitude internal waves, J. Fluid Mech., 380, 257-278, 1999.

Grue, J., Jensen, A., Rusås, P.-O., and Sveen, J. K.: Breaking and broadening of internal solitary waves, J. Fluid Mech., 413, 181217, 2000.

Helfrich, K. R. and Melville, W. K.: Long nonlinear internal waves, Annu. Rev. Fluid Mech., 38, 395-425, 2006.

Kakutani, T. and Yamasaki, N.: Solitary waves on a two-layer fluid, J. Phys. Soc. Jpn., 45, 674-679, 1978.

Lamb, H.: Hydrodynamics, 6th edn., Cambridge Univ. Press, Cambridge, UK, 1932.

Lannes, D. and Ming, M.: The Kelvin - Helmholtz instabilities in two-fluids shallow water models, Comm. Fields Inst., hal01101993, https://hal.archives-ouvertes.fr/hal-01101993 (last access: 11 January 2018), 2015.

Liapidevskii, V. Yu., Dutykh, D., and Gisclon, M.: On the modelling of shallow turbidity flows, Adv. Water Resources, 113, 310-327, 2018.

Long, R. R.: On the Boussinesq approximation and its role in the theory of internal waves, Tellus, 17, 46-52, 1965.

Makarenko, N., Maltseva, J., Tarakanov, R., and Ivanova, K.: Internal solitary waves in a layered weakly stratified flow, in: The Ocean in Motion, edited by: Velarde, M., Tarakanov, R., and Marchenko, A., Springer, Dordrecht, the Netherlands, 55-66, 2018.

Makarenko, N. I. and Maltseva, J. L.: An analytical model of large amplitude internal solitary waves, in: Extreme Ocean Waves, edited by: Pelinovsky, E. and Kharif, C., Springer, Dordrecht, the Netherlands, 179-189, 2008.

Makarenko, N. I. and Maltseva, J. L.: Phase velocity spectrum of internal waves in a weakly-stratified two-layer fluid, Fluid Dyn., 44, 278-294, 2009a.

Makarenko, N. I. and Maltseva, J. L.: Solitary waves in a weakly stratified two-layer fluid, J. Appl. Mech. Techn. Phys., 50, 229234, 2009b.

Miyata, M.: An internal solitary wave of large amplitude, La Mer, 23, 43-48, 1985.

Morozov, E., Demidov, A., Tarakanov, R., and Zenk, W.: Abyssal Channels in the Atlantic Ocean: Water Structure and Flows, Springer, Dordrecht, the Netherlands, 2010, 266 pp., https://doi.org/10.1007/978-90-481-9358-5, 2010.

Morozov, E. G.: Semidiurnal internal wave global field, Deep-Sea Res., 42, 135-148, 1995.

Morozov, E. G.: Oceanic internal tides, Observations, Analysis, and Modelling, Springer, Dordrecht, the Netherlands, 2018.

Morozov, E. G., Tarakanov, R. Yu., Lyapidevskii, V. Yu., and Makarenko, N. I.: Abyssal cataracts in the Romanche and Chain fracture zones, Dokl. Earth Sci., 446, 1211-1214, 2012. 
Ovsyannikov, L. V.: Two-layer shallow water model, J. Appl. Mech. Tech. Phys., 20, 127-135, 1979.

Ovsyannikov, L. V., Makarenko, N. I., Nalimov, V. I., Liapidevskii, V. V., Plotnikov, P. I., Sturova, I. V., Bukreev, V. I., and Vladimirov, V. A.: Nonlinear Problems of the Theory of Surface and Internal Waves, Nauka, Novosibirsk, Russia, 1985 (in Russian).

Tarakanov, R. Yu., Makarenko, N. I., and Morozov, E. G.: Antarctic bottom water flow in the western part of the Romanche Fracture Zone based on the measurements in October of 2011, Oceanology, 53, 655-667, https://doi.org/10.1134/S0001437013050147, 2013.
Thorpe, S. A.: Laboratory observations of secondary structures in Kelvin - Helmholtz billows and consequences for ocean mixing, Geophys. Astrophys. Fluid Dyn., 34, 175-190, 1985.

Turner, J. S.: Buoyancy effects in fluid, Cambridge Univ. Press, Cambridge, UK, 1973.

Van Haren, H., Gostiaux, L., Morozov, E., and Tarakanov, R.: Extremely long Kelvin - Helmholtz billow trains in the Romanche Fracture Zone, Geophys. Res. Lett., 44, 8445-8451, https://doi.org/10.1002/2014GL062421, 2014.

Voronovich, A. G.: Strong solitary internal waves in a 2.5 -layer model, J. Fluid Mech., 474, 85-94, 2003.

Yih, C. S.: Stratified flows, Academic Press, New York, USA, 1980. 